\begin{tabular}{|c|c|}
\hline Title & Mitochondrial targeting functional peptides as potential devices for the mitochondrial delivery of a DF-MITO-Porter \\
\hline Author(s) & Kawamura, Eriko; Y amada, Y uma; Harashima, Hideyoshi \\
\hline Citation & $\begin{array}{l}\text { Mitochondrion, 13(6), 610-614 } \\
\text { https:/doi.org/10.1016/.mito.2013.08.010 }\end{array}$ \\
\hline Issue Date & 2013-11 \\
\hline Doc URL & http:/hdl .handle.net/2115/56636 \\
\hline Type & article (author version) \\
\hline File Information & WoS_63683_Y amada.pdf \\
\hline
\end{tabular}

Instructions for use 


\title{
Mitochondrial targeting functional peptides as potential devices for the mitochondrial delivery of a DF-MITO-Porter
}

Eriko Kawamura ${ }^{1,2}$, Yuma Yamada ${ }^{1,2}$, Hideyoshi Harashima ${ }^{1,}$.

${ }^{1}$ Laboratory for Molecular Design of Pharmaceutics, Faculty of Pharmaceutical Sciences, Hokkaido University, Kita12, Nishi-6, Kita-ku, Sapporo 060-0812, Japan

${ }^{2}$ These authors contributed equally as first author

*To whom correspondence should be addressed: Laboratory for Molecular Design of Pharmaceutics, Faculty of Pharmaceutical Sciences, Hokkaido University, Kita-12, Nishi-6, Kita-ku, Sapporo 060-0812, Japan. E-mail: harasima@pharm.hokudai.ac.jp. Phone:+81-11-706-3919. Fax:+81-11-706-4879

\section{Keywords:}

Mitochondria, Mitochondrial drug delivery, Mitochondrial targeting peptide, MITO-Porter, S2 peptide, Nanoparticles

\begin{abstract}
To achieve mitochondrial therapy, we previously reported on the use of an octaarginine (R8) modified Dual Function (DF)-MITO-Porter for delivering molecules to mitochondria in living cells. In this study, using isolated mitochondria, homogenates and living cells, we evaluated the utility of mitochondrial targeting functional peptides as a ligand for delivering carriers. The S2 peptide modified carrier showed a high mitochondrial targeting activity in homogenates and living cells. In addition, the S2 peptide had a lower cell toxicity compared to R8 modified liposomes. The S2 peptide represents a potentially useful moiety for constructing an efficient and safe mitochondrial delivery system.
\end{abstract}




\section{Introduction}

To date, a number of investigators have reported that genetic defects in mitochondrial DNA are associated with mitochondrial diseases and a variety of human disorders, including neurodegenerative diseases, diabetes mellitus and cancer (Chan, 2006; Schapira, 2012; Taylor and Turnbull, 2005). An efficient mitochondrial targeting system and therapeutic molecules are required to accomplish mitochondrial gene therapy. The use of an endogenous mitochondrial targeting signal (MTS), cell penetrating peptides (CPPs), vesicles, and mitochondrial import proteins have been reported to be useful in delivering various cargoes, including low-molecular weight molecules, nucleic acids and proteins (Adhya et al., 2011; D’Souza et al., 2003; Flierl et al., 2003; Koczor et al., 2009; Mahata et al., 2006; Zhang et al., 2010). Nevertheless, a nanoparticle with a diameter that exceeds $100 \mathrm{~nm}$ has not been yet reported to accumulate in mitochondria efficiently compared to low-molecular weight molecules.

In a previous study, we reported on the development of a Dual Function (DF)-MITO-Porter, an innovative nanocarrier for achieving mitochondrial delivery, which has the ability to pass through the endosomal and mitochondrial membranes via step-wise membrane fusion (Yamada et al., 2011; Yamada et al., 2012a). In addition, we constructed the DF-MITO-Porter with oligodeoxynucleotide (oligo DNA) encapsulated, and estimated the intracellular multiple processes (Yamada et al., 2012b). The rate-limiting step for mitochondrial delivery was concluded to be the mitochondrial targeting process from the cytosol. Thus, to achieve mitochondrial therapy, it was clear that the carrier needed to be improved if it were to be useful as an optimal mitochondrial targeting system. The modified octaarginine (R8) has the ability to enhance cellular uptake and mitochondrial binding (Khalil et al., 2006; Yamada et al., 2008a), however we cannot exclude the possibility that it might interact with proteins in the cytosol since R8 is not selective for mitochondria.

Mitochondrial targeting peptides have been studied, in attempts to deliver certain molecular compounds to mitochondria. The R8 peptide, which mimics the trans-activating transcriptional activator (TAT), was used to modify the previously constructed MITO-Porter, and was found to function as a useful moiety for cellular uptake and mitochondrial targeting (Futaki et al., 2001; Khalil et al., 2006; Yamada et al., 2008a; Yamada et al., 2011). We also used the 31 amino acid sequence of the MTS derived from an endogenous protein (rat succinyl CoA synthetase) for delivering liposomes to mitochondria (Yamada et al., 2013). In constructing the MTS-MITO porter, aggregation was 
found to be a problem, and we failed to observe mitochondrial targeting activity of MTS-MITO-Porter in living cells. However, inserting polyethylene glycol (PEG) as a linker between the MTS and lipids enabled the preparation of an alternate type of MTS-MITO-Porter (Kawamura et al., 2013). Although the constructed DF-MTS-MITO-Porter showed an enhanced cellular uptake, intracellular trafficking was not greatly improved. We hypothesized that the limited trafficking of the carriers was due to the tendency of the long sequence of MTS to undergo aggregation within cells.

Based on these results, we focused on mitochondrial targeting functional peptides with shorter sequences (Yamada et al., 2008b). Szeto and Schiller (2011) reported on the development of a Szeto-Schiller (SS) peptide, a cell permeable antioxidant, in which aromatic residues alternated with basic amino acids. This peptide was reported to be predominantly concentrated in mitochondria within cells (Zhao et al., 2004). Wipf et al. (2005) reported on a conjugate of 4-amino-TEMPO and gramicidin S (GS) segment (XJB-5-131), a mitochondria targeted electron and ROS scavenger (Fink et al., 2007). Mitochondrial penetrating peptides (MPPs) contain repeating sequences of positively charged arginine residues and lipophilic cyclohexylalanine and are designed to exhibit efficient cellular uptake and to accumulate in the mitochondrial matrix (Horton et al., 2008; Horton et al., 2012). The circular peptide (CP) is known to associate with prohibitin in adipose tissue (Hossen et al., 2010; Kolonin et al., 2004), and would be a useful moiety for mitochondrial targeting because the mitochondrial membrane contains prohibitin. We report herein on an investigation of whether the mitochondrial targeting activity of nanocarriers can be enhanced in a similar manner to R8 by modifying a mitochondrial targeting peptide. 


\section{Materials and methods}

\subsection{Materials}

1, 2-dioleoyl-sn-glycero-3-phosphatidyl ethanolamine (DOPE), sphingomyelin (SM) and rhodamine-DOPE were purchased from Avanti Polar lipids (Alabaster, AL, USA). Phosphatidic acid (PA) and cholesteryl hemisuccinate (CHEMS) were obtained from Sigma-Aldrich (St. Louis, MO, USA). Stearyl R8 (STR-R8) and cholesteryl-GALA (cholesteryl-WEAALAEALAEALAEHLAEALAEALEALAA-NH ${ }_{2}$ ) were obtained from Kurabo Industries Ltd (Osaka, Japan). STR-SS (stearyl-Dmt- $D$-Arg-FK-NH 2 , Dmt=2, 6-dimethyltyrosine), STR-S2 (stearyl-Dmt- $D^{-A r g-F K-~}$ Dmt- $D$-Arg-FK-NH 2 )，STR-GS (L- $D$-Phe-PV-Orn-(K)-Stearoyl)，STR-CP (GKGGRAKDGG(K)-Stearoyl) were obtained from Toray Research Center (Tokyo, Japan). STR-MPP6 (stearyl-XRXRXR-OH, X=cyclohexylalanine) was purchased from Sigma-Aldrich (St. Louis, MO, USA). HeLa human cervix carcinoma cells were purchased from the ATCC (Manassas, VA, USA). Dulbecco's modified Eagle medium (DMEM), fetal bovine serum (FBS) and Rhodamine123 were purchased from Invitrogen Corporation (Carlscad, CA, USA). The oligo DNA (5'CTTCTCGTCCCCATGGATGACCCC-3') and the oligo DNA labeled with fluorescent that Cy5 is conjugated to 5 terminal of the oligo DNA were obtained from Hokkaido System Science Co., Ltd (Sapporo, Japan). All other chemicals used were commercially available reagent-grade products.

\subsection{Construction of mitochondrial targeting peptide modified liposomes}

Liposomes modified with mitochondrial targeting peptides were constructed by lipid film hydration method. A lipid film was formed by evaporating a chloroform solution of 137.5 nmol lipid [DOPE/SM (9:2, molar ratio)] containing each peptide-lipid conjugate [STR-R8, SS, S2, MPP6, GS and CP (10 and 15 mol\% of lipid)]. After the formation of the film, $250 \mu \mathrm{l}$ of $10 \mathrm{mM}$ HEPES buffer was added, followed by incubation for 15 min and sonication for $30 \mathrm{sec}$ in a bath-type sonicator. The particle diameter was measured by a quasi-elastic light scattering method, and the $\zeta$ potential was determined electrophoretically by means of an electrophoretic light scattering spectrophotometer (Zetasizer Nano ZS; Malvern Instruments, Herrenberg, Germany). 


\subsection{Mitochondrial binding activity using isolated mitochondria}

The mitochondrial binding activity of the peptide modified liposome was determined by fluorescence measurements. Mitochondria were isolated from livers obtained from adult male wistar rats (6-8 weeks old) as previously reported (Yamada et al., 2008a). A suspension of isolated mitochondria $80 \mu \mathrm{l}(1 \mathrm{mg} \operatorname{protein} / \mathrm{ml})$ and a rhodamine labeled liposome $20 \mu \mathrm{l}$ (lipid concentration $0.55 \mathrm{mM}$ ) were mixed and incubated for $30 \mathrm{~min}$ at $25^{\circ} \mathrm{C}$. The resulting solution was denoted as sample $A$. The suspension was centrifuged $\left(16,000 \mathrm{~g}, 10 \mathrm{~min}, 4^{\circ} \mathrm{C}\right)$, and washed twice with mitochondrial isolation buffer (MIB) and re-precipitated by centrifugation $\left(24,000 \mathrm{~g}, 2 \mathrm{~min}, 4^{\circ} \mathrm{C}\right)$. The pellet was resuspended in $100 \mu \mathrm{l}$ MIB to give sample B. Samples A and B were dissolved in SDS (final concentration 0.5\%) and the fluorescence intensities were measured with excitation at $557 \mathrm{~nm}$ and emission at $571 \mathrm{~nm}$ (EnSpireTM 2300 Multilabel Reader; PerkinElmer Inc. Waltham, MA, USA).

Mitochondrial binding activity was calculated as follows:

Mitochondrial binding activity $(\%)=\mathrm{F}_{\mathrm{B}} / \mathrm{F}_{\mathrm{A}} \times 100$

where $\mathrm{F}_{\mathrm{A}}$ and $\mathrm{F}_{\mathrm{B}}$ represent the fluorescence intensity of sample $\mathrm{A}$ and $\mathrm{B}$.

\subsection{Mitochondrial targeting activity using rat liver homogenate}

Rats were sacrificed and phosphate-buffered saline (PBS (-)) was injected into the portal vein after cutting the vena cana to remove the remaining blood in the livers. Livers were excised and placed in ice cold MIB. All subsequent steps were carried out on ice. The livers were then minced into small pieces and the suspension was homogenized in a glass homogenizer with a pestle (approximately $550 \mathrm{rpm}$ ). The homogenate was diluted with MIB (final concentration $10 \% \mathrm{w} / \mathrm{v}$ ) and filtered through nylon meshe. $1 \mathrm{~mol} \%$ rhodamine labeled liposome $60 \mu \mathrm{l}$ (lipid concentration $0.55 \mathrm{mM}$ ) was added to the prepared homogenate solution $540 \mu \mathrm{l}(5 \mathrm{mg}$ protein $/ \mathrm{ml})$, and incubated for $30 \mathrm{~min}$ at $25^{\circ} \mathrm{C}$. The resulting solution was denoted as sample A. Each organelle fraction, nucleus, mitochondria, cytosol and other was obtained from the homogenate using differential centrifugation. The suspension was centrifuged at $700 \mathrm{~g}$ for $10 \mathrm{~min}$ at $4{ }^{\circ} \mathrm{C}$ to precipitate the nuclei. The supernatant was removed and centrifuged at $16,000 \mathrm{~g}$ for $10 \mathrm{~min}$ at $4{ }^{\circ} \mathrm{C}$ to precipitate 
mitochondria. The supernatant was removed and centrifuged at $20,400 \mathrm{~g}$ for $10 \mathrm{~min}$ at $4{ }^{\circ} \mathrm{C}$, and the resulting supernatant was used as the cytosol fraction. The pelleted fractions were resuspended in $100 \mu 1$ of MIB. Sample A and all fractions were dissolved in $100 \mu \mathrm{l}$ of $1 \%$ SDS solution and the fluorescence intensities were measured (Ex $470 \mathrm{~nm} / \mathrm{Em} 530 \mathrm{~nm}$ ). The targeting activity (\%) was calculated as follows:

Targeting activity $(\%)=\mathrm{F} / \mathrm{F}_{\mathrm{A}} \times 100$

where $\mathrm{F}_{\mathrm{A}}$ and $\mathrm{F}$ represent the fluorescence intensity of sample $\mathrm{A}$ and each homogenate fractions, nucleus, mitochondria, cytosol and other.

\subsection{Evaluation of cell toxicity using MTT assay}

HeLa cells $\left(4 \times 10^{4}\right.$ cells) were seeded on a 24 -well plate (Corning) with DMEM, containing $10 \%$ FBS, under an atmosphere of $5 \% \mathrm{CO}_{2} /$ air at $37{ }^{\circ} \mathrm{C}$ for $24 \pm 3 \mathrm{~h}$. The cells were washed with PBS (-) before incubation with the carriers (final concentrations of lipid, $0.0275,0.055,0.11,0.22 \mathrm{mM}$ ). The cells were then incubated in serum-free medium for $1 \mathrm{~h}$ under an atmosphere of $5 \% \mathrm{CO}_{2} /$ air at $37^{\circ} \mathrm{C}$. After replacing the medium with fresh DMEM containing $10 \%$ serum, the cells were incubated for an additional $2 \mathrm{~h}$. Cell viabilities were measured using a Cell Proliferation Assay System with a Tetra Color ONE (Seikagaku Biobusiness, Tokyo, Japan). In brief, tetrazolium (WST-8) was added to a final volume of $275 \mu \mathrm{l} /$ well. After 1-h incubation, the absorbance at $450 \mathrm{~nm}$ was measured.

Cell viability was calculated as follows:

Cell viability $=\mathrm{A}_{\mathrm{T}} / \mathrm{A}_{\mathrm{U}}$

where $A_{T}$ and $A_{U}$ represent the absorbance at $450 \mathrm{~nm}$ for treated and untreated cells, respectively.

\subsection{Intracellular observation of DF-MITO-Porter using CLSM}

The oligo DNA encapsulated DF-R8-MITO-Porter and DF-S2-MITO-Porter was constructed by multi-layering method. The solutions of oligo DNA $(0.1 \mathrm{mg} / \mathrm{ml}, 10 \mathrm{~mol} \%$ Cy5 labeled $)$ and STR-R8 were mixed under vortexing at a nitrogen /phosphate ratio of 3. The constructed nanoparticle of oligo DNA and STR-R8 peptide were encapsulated in the mitochondrial fusogenic lipid [DOPE/SM/CHEMS (9:2:1, molar ratio)] by the lipid film hydration method (Yamada 
et al., 2008a). STR-R8, S2 were added to the solution (10 mol\% of lipid), and conventional R8-MITO-Porter, S2MITO-Porter were constructed. R8-MITO-Porter and S2-MITO-Porter were further coated with endosomal fusogenic lipid [DOPE/PA/STR-R8/Chol-GALA (7:2:1:0.1, molar ratio)] to construct the DF-R8-MITO-Porter, and the DF-S2MITO-Porter (El-Sayed et al., 2008). Intracellular trafficking was observed by confocal laser scanning microscopy (CLSM) as previously reported (Yamada et al., 2012b). HeLa cells $\left(4 \times 10^{4}\right.$ cells) were cultured in $35 \mathrm{~mm}$ dishes (IWAKI) with DMEM, which contained $10 \% \mathrm{FBS}$, under an atmosphere of $5 \% \mathrm{CO}_{2} /$ air at $37^{\circ} \mathrm{C}$ for $24 \pm 3 \mathrm{~h}$. The DFS2-MITO-Porter or the DF-R8-MITO-Porter was added to the HeLa cells (final concentration of oligo DNA, $80 \mathrm{nM}$ ). The cells were then incubated in phenol red-free medium without serum under atmosphere of $5 \% \mathrm{CO}_{2} /$ air at $37^{\circ} \mathrm{C}$. After 1-h incubation, the medium was replaced with fresh phenol red-free medium containing serum, and the cells were incubated for $2 \mathrm{~h}$. The medium was replaced with fresh medium containing Rhodamine123 (final concentration, 100 $\mathrm{ng} / \mathrm{mL}) 30 \mathrm{~min}$ before the acquisition of the fluorescence images, and the cells were incubated in this solution. After this incubation, the cells were washed with the phenol red-free medium containing serum, and then observed by CLSM (FV10i-LIV; Olympus Corporation, Tokyo, Japan). The cells were excited with a $473 \mathrm{~nm}$ light for detecting Rhodamine123 and a $635 \mathrm{~nm}$ light for detecting Cy5 from a LD laser. Images were obtained using a FV10i-LIV equipped with a water immersion objective lens (UPlanSApo 60x/NA $=1.2$ ) and a diachronic mirror (DM 405/473/559/635). The two fluorescence detection Chs were set to the following filters: Ch1: BP 465-515 (red pseudo color) for Rhodamine123 and Ch2: BP 610-710 (green pseudo color) for Cy5.

\section{Results and discussion}

3.1. Construction of liposomes modified with mitochondrial targeting peptides and an evaluation of mitochondrial binding activity

Mitochondrial targeting peptides, R8, SS, GS, MPP and CP were used to prepare the mitochondrial targeting carriers. We also synthesized a S2 peptide by conjugating two SS peptide sequences, assuming that the mitochondrial targeting ability of the peptide would be enhanced. The liposomes [DOPE/SM (9:2, molar ratio)] were constructed by adding the stearyl peptides at levels of 10 or $15 \%$ of the total lipid using the lipid hydration method. MPP 6 , a sequence 
of six amino acid residues of MPP, was used in constructing the carrier because peptides with more than eight amino acids of the MPP sequence tend to aggregate when liposomes are modified with them (data not shown). Table 1 shows the diameter, polydispersity index (PDI) and $\zeta$ potential of the constructed carriers. All the carriers had diameters of approximately $100 \mathrm{~nm}$, and the $\zeta$ potentials of the unmodified and modified carriers were approximately $-30 \mathrm{mV}$ and $40 \mathrm{mV}$ respectively. The fact that the surface charge was changed indicates that the liposome was successfully modified with each peptide. We next evaluated the mitochondrial binding activity of the constructed carriers. Rhodamine labeled liposomes were incubated with isolated mitochondria and their mitochondrial binding activities measured (Table 1). The binding activity of unmodified liposomes was negligible (3\%), however all of peptides showed a high mitochondrial binding activity of approximately $90 \%$. This suggests that the peptide was displayed on the surface of the liposome, and functioned as a ligand to bind the carrier to mitochondria.

\subsection{Mitochondrial targeting activity}

To verify the mitochondrial targeting activity of the peptide modified liposomes in an intracellular environment, a rat liver homogenate was used as a model system. Liposomes labeled with a fluorescent dye were incubated with the prepared homogenate, and the targeting activities of the carriers in each fraction, nucleus, mitochondria, cytosol and others were measured. Based on the mitochondrial binding activities shown in Table. 1, it was assumed that the surface electric charge of the liposome affected the mitochondrial targeting activity similarly, and all peptides would be expected to show a high value. However, only the S2 peptide showed a high mitochondrial targeting activity, which was nearly equal to that for the conventional R8 (Fig. 1A), approximately $40 \%$ for the R8 and S2 peptide modified liposomes (15\% modification). The mitochondrial targeting activities of other peptides were comparable with that for an unmodified liposome, and most of the liposomes remained in the cytosol fraction (Fig. S1). We then investigated factors that could affect the targeting activity of the carrier in the homogenate. The mitochondrial targeting activity of liposomes that were modified with $15 \%$ peptide and their cationic valences were plotted in a graph (Fig. 1B). The results show that mitochondrial targeting activity was significantly correlated with the cationic valence of each peptide. These results suggest that a nanocarrier requires a certain cationic valence for it to be delivered to mitochondria in a homogenate. 


\subsection{Cell viability and intracellular observation of DF-MITO-Porter}

To compare the utility of the R8 and S2 peptide modified liposome for mitochondrial targeting, the cell viability of each carrier was investigated. Unmodified liposomes, the 15\% R8 and S2 peptide modified liposomes were added to HeLa cells in different lipid concentrations, and cell viability was measured using an MTT assay after a 3-h incubation (Fig. 2). The cell viability of the R8 peptide decreased dramatically with increasing lipid concentration. On the other hand, no detectable cell toxicity was observed for the unmodified and S2 peptide modified liposomes. The addition of a high dose of R8 modified liposomes, however, induced cytotoxicity. It can therefore be concluded that the S2 peptide represents a safer targeting moiety than R8 for mitochondrial delivery.

We observed the intracellular trafficking of the encapsulated oligo DNA in DF-S2-MITO-Porter by CLSM to evaluate the mitochondrial targeting activity of the S2 peptide in living cells. A Cy5 labeled oligo DNA nanoparticle was encapsulated in S2 peptide modified liposomes [DOPE/SM/CHEMS (9:2:1, molar ratio)], and the carrier was further coated with an endosomal fusogenic envelope [DOPE/PA/STR-R8/chol-GALA (7:2:1:0.1, molar ratio)] comprised of a cellular uptake moiety (R8) and an endosomal escape moiety (GALA) constructed by the multi-layering method (Akita et al., 2009; Akita et al., 2011; El-Sayed et al., 2008; Futaki et al., 2005; Yamada et al., 2012b). A number of nanoparticles were observed to accumulate around the nucleus where most of the mitochondria are located (Fig. 3). Since cellular uptake is dependent on the R8 being modified to the outermost layer of the DF-MITO-Porter, it can be concluded that the cellular uptake efficiency of the DF-S2-MITO-Porter and DF-R8-MITO-Porter is the same, however the intracellular trafficking would be different. The DF-S2-MITO-Porter appeared to be dispersed in the cytosol, whereas the DF-R8-MITO-Porter aggregated in the cytosol and showed a stronger fluorescence intensity.

Both the DF-R8-MITO-Porter and DF-S2-MITO-Porter (green pseudo color) were colocalized with mitochondria (red pseudo color), and observed as a yellow signal suggesting that the carriers were delivered to the mitochondria in living cells. In a previous study, we showed that the DF-MITO-Porter successfully delivered a low molecular weight molecule and DNase I protein to the mitochondrial matrix in living cells (Yamada et al., 2011; Yasuzaki et al., 2010). Based on these results, we consider that the DF-S2-MITO-Porter is functionally capable of delivering its content to mitochondria in living cells. Attempts to verify the delivery of nanoparticles to the 
mitochondrial matrix are currently underway.

\section{Conclusion}

A number of researchers have reported that synthesized mitochondrial targeting peptides can associate with mitochondria in living cells by electrostatic interactions or mitochondrial affinity (Fink et al., 2007; Horton et al., 2008; Szeto and Schiller, 2011). However, the use of these peptides did not show a similar behavior when they were used as modifiers of nanoparticles. An evaluation of mitochondrial targeting activity using a homogenate demonstrated that mitochondrial targeting activity of the carrier is dependent on the cationic valence of the peptide, and a certain balance of positive charge and hydrophobicity is required for the successful binding of a liposome to a mitochondrion. The difference in the mitochondrial targeting activity of the peptides themselves and when they are modified to the liposome can be attributed to differences in their sizes and fluidities in the cytosol.

The surfaces of liposomes were successfully modified with mitochondrial targeting peptides without aggregation, unlike the previously used MTS. All the peptides functioned as ligands to bind the carrier to the mitochondria. In addition, the S2 peptide possessed a low cell toxicity compared to R8 while the mitochondrial targeting activity in a homogenate and living cells were similar to that for R8. The findings reported herein indicate that the S2 peptide represents a potentially ideal modifier for a mitochondrial targeting nanocarrier. Studies related to this issue are currently in progress.

\section{Acknowledgments}

This work was supported, in part by, the Program for Promotion of Fundamental Studies in Health Sciences of the National Institute of Biomedical Innovation, Japan (NIBIO), a Grant-in-Aid for Young Scientists (A) and a Grantin-Aid for Scientific Research (S) from the Ministry of Education, Culture, Sports, Science and Technology of Japanese Government (MEXT). We also thank Milton Feather for his helpful advice in writing the manuscript.

\section{References}


Adhya, S., Mahato, B., Jash, S., Koley, S., Dhar, G., Chowdhury, T., 2011. Mitochondrial gene therapy: The tortuous path from bench to bedside. Mitochondrion 11, 839-844.

Akita, H., Kudo, A., Minoura, A., Yamaguti, M., Khalil, I.A., Moriguchi, R., Masuda, T., Danev, R., Nagayama, K., Kogure, K., Harashima, H., 2009. Multi-layered nanoparticles for penetrating the endosome and nuclear membrane via a step-wise membrane fusion process. Biomaterials 30, 2940-2949.

Akita, H., Kogure, K., Moriguchi, R., Nakamura, Y., Higashi, T., Nakamura, T., Serada, S., Fujimoto, M., Naka, T., Futaki, S., Harashima, H., 2011. Reprint of: Nanoparticles for ex vivo siRNA delivery to dendritic cells for cancer vaccines: Programmed endosomal escape and dissociation. J. Control. Release 149, 58-64.

Chan, D.C., 2006. Mitochondria: Dynamic Organelles in Disease, Aging, and Development. Cell 125, 1241-1252.

D’Souza, G.G.M., Rammohan, R., Cheng, S.M., Torchilin, V.P., Weissig, V., 2003. DQAsome-mediated delivery of plasmid DNA toward mitochondria in living cells. J. Control. Release 92, 189-197.

El-Sayed, A., Khalil, I.A., Kogure, K., Futaki, S., Harashima, H., 2008. Octaarginine- and octalysine-modified nanoparticles have different modes of endosomal escape. J. Biol. Chem. 283, 23450-23461.

Fink, M.P., Macias, C.A., Xiao, J., Tyurina, Y.Y., Jiang, J., Belikova, N., Delude, R.L., Greenberger, J.S., Kagan, V.E., Wipf, P., 2007. Hemigramicidin-TEMPO conjugates: Novel mitochondria-targeted anti-oxidants. Biochem. Pharmacol. 74, 801-809.

Flierl, A., Jackson, C., Cottrell, B., Murdock, D., Seibel, P., Wallace, D.C., 2003. Targeted Delivery of DNA to the Mitochondrial Compartment via Import Sequence-Conjugated Peptide Nucleic Acid. Mol. Ther. 7, 550-557.

Futaki, S., Ohashi, W., Suzuki, T., Niwa, M., Tanaka, S., Ueda, K., Harashima, H., Sugiura, Y., 2001. Stearylated arginine-rich peptides: a new class of transfection systems. Bioconjug. Chem. 12, 1005-1011.

Futaki.,S., Masui, Y., Nakase, I., Sugiura, Y., Nakamura, T., Kogure, K., Harashima, H., 2005. Unique features of a pHsensitive fusogenic peptide that improves the transfection efficiency of cationic liposomes. J. Gene Med. 7, 14501458.

Horton, K.L., Stewart, K.M., Fonseca, S.B., Guo, Q., Kelley, S.O., 2008. Mitochondria-Penetrating Peptides. Chem. Biol. 15, 375-382.

Horton, K.L., Pereira, M.P., Stewart, K.M., Fonseca, S.B., Kelley, S.O., 2012. Tuning the Activity of MitochondriaPenetrating Peptides for Delivery or Disruption. ChemBioChem 13, 476-485.

Hossen, M.N., Kajimoto, K., Akita, H., Hyodo, M., Ishitsuka, T., Harashima, H., 2010. Ligand-based targeted delivery of a peptide modified nanocarrier to endothelial cells in adipose tissue. J. Control. Release 147, 261-268.

Kawamura, E., Yamada, Y., Yasuzaki, Y., Hyodo, M., Harashima, H., 2013. Intracellular observation of nanocarriers modified with a mitochondrial targeting signal peptide. J. Biosci. Bioeng. doi: 10.1016/j.jbiosc.2013.05.001.

Khalil, I.A., Kogure, K., Futaki, S., Harashima, H., 2006. High density of octaarginine stimulates macropinocytosis leading to efficient intracellular trafficking for gene expression. J. Biol. Chem. 281, 3544-3551.

Koczor, C.A., Snyder, J.W., Shokolenko, I.N., Dobson, A.W., Wilson, G.L., Ledoux, S.P., 2009. Targeting Repair Proteins to the Mitochondria of Mammalian Cells Through Stable Transfection, Transient Transfection, Viral Transduction, and TAT-Mediated Protein Transduction. Methods Mol. Biol. 554, 233-249.

Kolonin, M.G., Saha, P.K., Chan, L., Pasqualini, R., Arap, W., 2004. Reversal of obesity by targeted ablation of adipose tissue. Nat. Med. 10, 625-632.

Mahata, B., Mukherjee, S., Mishra, S., Bandyopadhyay, A., Adhya, S., 2006. Functional Delivery of a Cytosolic tRNA into Mutant Mitochondria of Human Cells. Science 314, 471-474.

Schapira, A.H.V., 2012. Mitochondrial disease. Lancet 379, 1825-1834.

Shokolenko, I.N., Alexeyev, M.F., LeDoux, S.P., Wilson, G.L., 2005. TAT-mediated protein transduction and targeted delivery of fusion proteins into mitochondria of breast cancer cells. DNA Repair 4, 511-518.

Szeto, H.H., Schiller, P.W., 2011. Novel Therapies Targeting Inner Mitochondrial Membrane-From Discovery to Clinical Development. Pharm. Res. 28, 2669-2679.

Taylor, R.W., Turnbull, D.M., 2005. Mitochondrial DNA Mutations in Human Disease. Nat. Rev. Genet. 6, 389-402.

Wipf, P., Xiao, J., Jiang, J., Belikova, N.A., Tyurin, V.A., Fink, M.P., Kagan, V.E., 2005. Mitochondrial Targeting of Selective Electron Scavengers: Synthesis and Biological Analysis of Hemigramicidin-TEMPO Conjugates. J. Am. Chem. Soc. 127, 12460-12461.

Yamada, Y., Akita, H., Kamiya, H., Kogure, K., Yamamoto, T., Shinohara, Y., Yamashita, K., Kobayashi, H., Kikuchi, H., Harashima, H., 2008a. MITO-Porter: A liposome-based carrier system for delivery of macromolecules into mitochondria via membrane fusion. Biochim. Biophys. Acta 1778, 423-432.

Yamada, Y., Harashima, H., 2008b. Mitochondrial drug delivery systems for macromolecule and their therapeutic 
application to mitochondrial diseases. Adv. Drug Deliv. Rev. 60, 1439-1462.

Yamada, Y., Furukawa, R., Yasuzaki, Y., Harashima, H., 2011. Dual Function MITO-Porter, a Nano Carrier Integrating Both Efficient Cytoplasmic Delivery and Mitochondrial Macromolecule Delivery. Mol. Ther. 19, 1449-1456.

Yamada, Y., Harashima, H., 2012a. Delivery of bioactive molecules to the mitochondrial genome using a membranefusing, liposome-based carrier, DF-MITO-Porter. Biomaterials 33, 1589-1595.

Yamada, Y., Kawamura, E., Harashima, H., 2012b. Mitochondrial-targeted DNA delivery using a DF-MITO-Porter, an innovative nano carrier with cytoplasmic and mitochondrial fusogenic envelopes. J. Nanopart. Res. 14, 1013.

Yamada, Y., Harashima, H., 2013. Enhancement in selective mitochondrial association by direct modification of a mitochondrial targeting signal peptide on a liposomal based nanocarrier. Mitochondrion. doi: 10.1016/j.mito.2012.09.001.

Yasuzaki, Y., Yamada, Y., Harashima, H., 2010. Mitochondrial matrix delivery using MITO-Porter, a liposome-based carrier that specifies fusion with mitochondrial membranes. Biochem. Biophys. Res. Commun. 397, 181-186.

Zhang, E., Zhang, C., Su, Y., Cheng, T., Shi, C., 2011. Newly developed strategies for multifunctional mitochondriatargeted agents in cancer therapy. Drug Discov. Today 16, 140-146.

Zhao, K., Zhao, G.M., Wu, D., Soong, Y., Birk, A.V., Schiller, P.W., Szeto, H.H., 2004. Cell-permeable Peptide Antioxidants Targeted to Inner Mitochondrial Membrane inhibit Mitochondrial Swelling, Oxidative Cell Death, and Reperfusion Injury. J. Biol. Chem. 279, 34682-34690.

\section{Figure legends}

Fig. 1. Mitochondrial targeting activities of carriers. (A) Mitochondrial targeting activity (\%) of unmodified liposomes (gray bar), 10\% (filled bars) and 15\% (open bars) peptide modified liposome in rat liver homogenate solution. Data are mean \pm S.D. $(n=3-5)$. Significant differences between unmodified liposome and peptide modified liposomes $(* * \mathrm{p}<0.01$ by one-way ANOVA, followed by Bonferroni test). (B) Correlation between mitochondrial targeting activities (\%) of R8 (filled diamond), SS (open triangle), S2 (filled rectangle), MPP6 (filled triangle), GS (filled circle), CP (open diamond) modified liposomes and cation valences (correlation coefficient $r=0.91, \mathrm{p}<0.05$ significant correlation).

Fig. 2. Evaluation of cell viability. Cell viabilities of unmodified liposomes (circles), 15\% R8 (triangles) and S2 (squares) peptide modified liposomes were measured at different lipid concentrations $(0.0275,0.055,0.11,0.22 \mathrm{mM})$. Cell viability was evaluated by the following equation; Cell viability $=A_{T} / A_{U}$, where $A_{T}$ and $A_{U}$ represent the absorbance at $450 \mathrm{~nm}$ for treated and untreated cells, respectively. Data are represented by the mean \pm S.D. $(n=3)$.

Fig. 3. Intracellular observation of carriers. Intracellular observation of co-localization between Cy5 labeled oligo DNA (green pseudo color) encapsulated DF-S2-MITO-Porter (A), DF-R8-MITO-Porter (B) and mitochondria stained with Rhodamine123 (red pseudo color) using CLSM. The carriers (green pseudo color) were observed to co-localize with mitochondria (red pseudo color), observed as yellow signals in the merged images. Scale bars $10 \mu \mathrm{m}$. 
Fig. 1

A

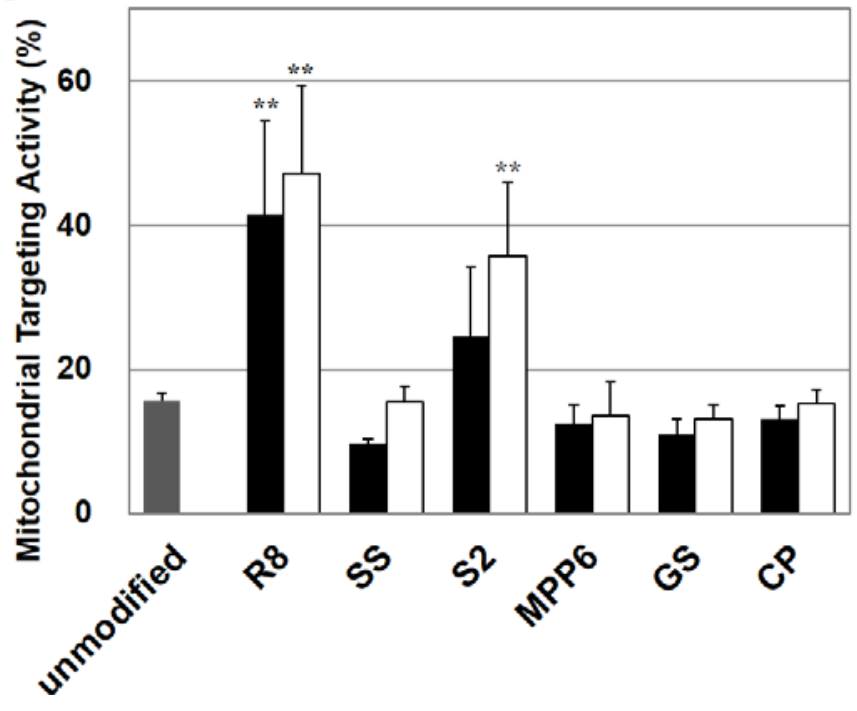

B

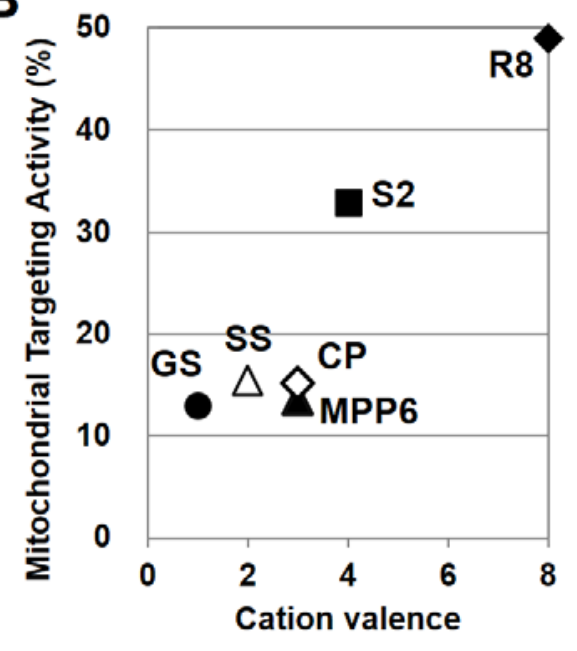


Fig. 2

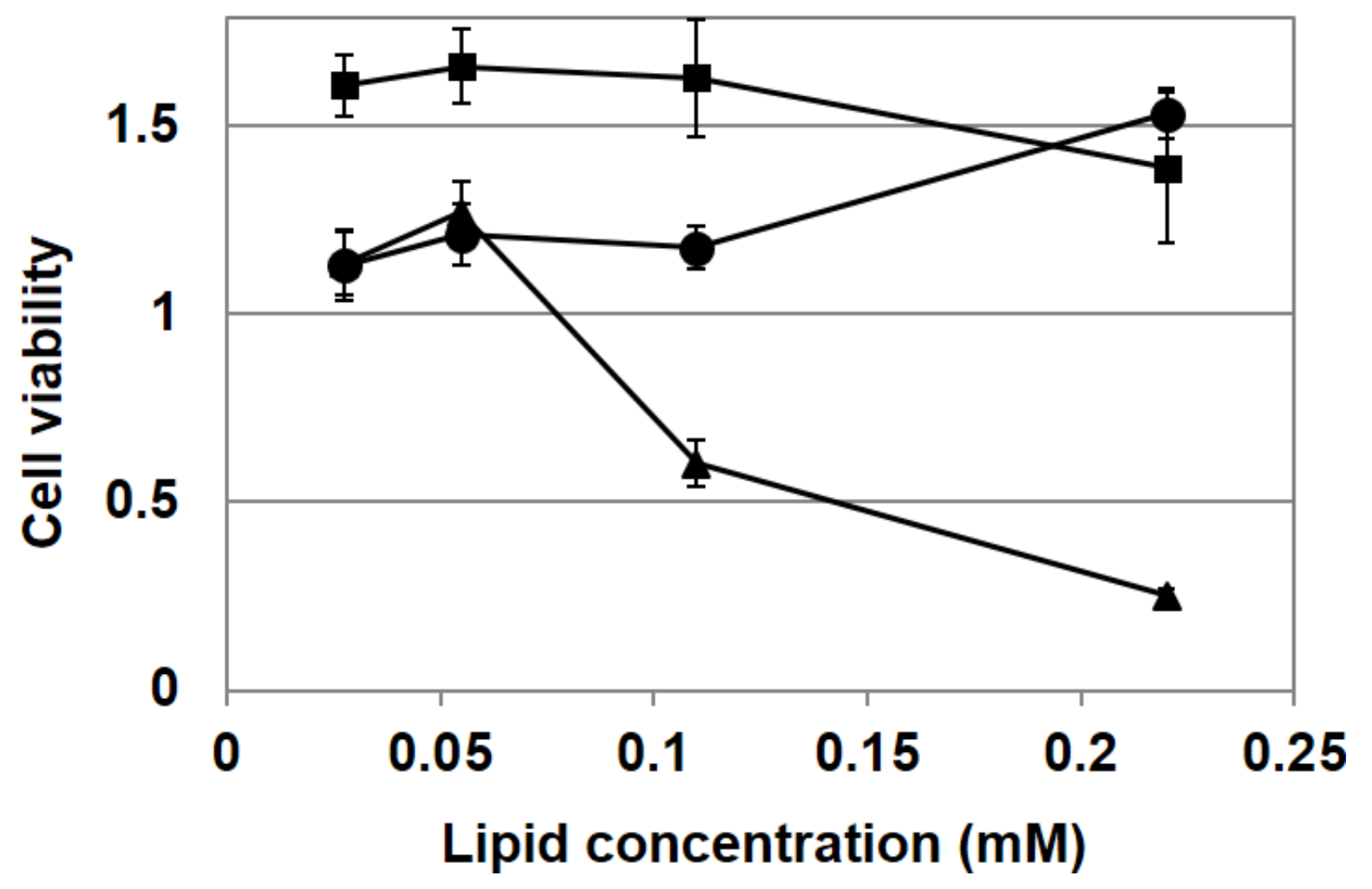


Fig. 3

A

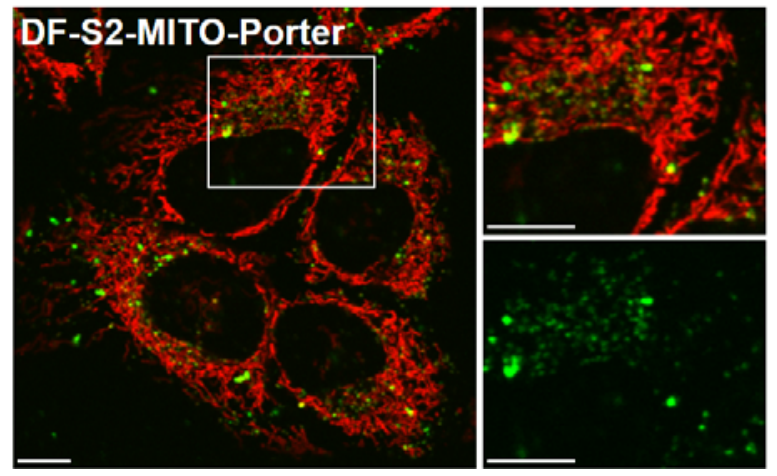

B

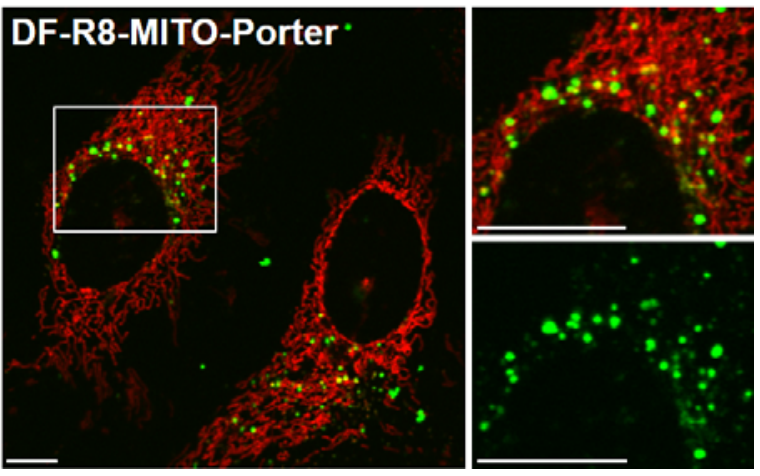




\section{Table 1}

Physicochemical properties and mitochondrial binding activities of the carriers

\begin{tabular}{|c|c|c|c|c|c|c|c|}
\hline Peptide & Sequence & Properties of peptides & $\begin{array}{l}\text { Modification } \\
\text { rate }(\%)\end{array}$ & $\begin{array}{l}\text { Diameter } \\
(\mathrm{nm})\end{array}$ & PDI & $\begin{array}{l}\zeta \text { potential } \\
(\mathrm{mV})\end{array}$ & $\begin{array}{c}\text { Mitochondrial } \\
\text { binding activity (\%) }\end{array}$ \\
\hline Unmodified & - & - & - & $106 \pm 25$ & $0.254 \pm 0.02$ & $-29 \pm 5$ & $3 \pm 0.5$ \\
\hline \multirow{2}{*}{ R8 } & \multirow{2}{*}{ RRRRRRRR } & \multirow{2}{*}{$\begin{array}{c}\text { Cell permeable } \\
\text { (Futaki et al., 2001) }\end{array}$} & 10 & $93 \pm 9$ & $0.227 \pm 0.01$ & $48 \pm 4$ & $84 \pm 9$ \\
\hline & & & 15 & $90 \pm 6$ & $0.239 \pm 0.03$ & $45 \pm 8$ & $100 \pm 14$ \\
\hline \multirow{2}{*}{ ss } & \multirow{2}{*}{ Dmt-o-Arg-FK } & \multirow{4}{*}{$\begin{array}{l}\text { Cell permeable antioxidant } \\
\text { (Szeto and Schiller, 2011) }\end{array}$} & 10 & $101 \pm 10$ & $0.243 \pm 0.01$ & $34 \pm 4$ & $79 \pm 9$ \\
\hline & & & 15 & $95 \pm 10$ & $0.252 \pm 0.03$ & $41 \pm 5$ & $90 \pm 6$ \\
\hline \multirow{2}{*}{ s2 } & \multirow{2}{*}{$\begin{array}{l}\text { Dmt-o-Arg-FK- } \\
\text { Dmt-o-Arg-FK }\end{array}$} & & 10 & $95 \pm 14$ & $0.247 \pm 0.02$ & $46 \pm 8$ & $87 \pm 10$ \\
\hline & & & 15 & $89 \pm 6$ & $0.240 \pm 0.03$ & $51 \pm 7$ & $100 \pm 11$ \\
\hline \multirow{2}{*}{ MPP6 } & \multirow{2}{*}{ XRXRXR } & \multirow{2}{*}{$\begin{array}{l}\text { Cell permeable, positive } \\
\text { charge and hydrophobicity } \\
\text { (Horton et al., 2008) }\end{array}$} & 10 & $103 \pm 11$ & $0.232 \pm 0.01$ & $38 \pm 5$ & $78 \pm 9$ \\
\hline & & & 15 & $102 \pm 11$ & $0.259 \pm 0.04$ & $43 \pm 5$ & $92 \pm 10$ \\
\hline \multirow{2}{*}{ GS } & \multirow{2}{*}{ L-o-Phe-PV-Orn } & \multirow{2}{*}{$\begin{array}{l}\text { Fragment of gramicidin S } \\
\text { (Wipf et al., 2005) }\end{array}$} & 10 & $107 \pm 5$ & $0.350 \pm 0.04$ & $31 \pm 7$ & $80 \pm 8$ \\
\hline & & & 15 & $101 \pm 14$ & $0.268 \pm 0.12$ & $30 \pm 15$ & $92 \pm 8$ \\
\hline \multirow{2}{*}{ CP } & \multirow{2}{*}{ GKGGRAKDGG } & \multirow{2}{*}{$\begin{array}{l}\text { Affinity to prohibitin } \\
\text { (Kolonin et al., 2004) }\end{array}$} & 10 & $109 \pm 6$ & $0.325 \pm 0.03$ & $42 \pm 2$ & $77 \pm 8$ \\
\hline & & & 15 & $96 \pm 6$ & $0.299 \pm 0.09$ & $37 \pm 10$ & $87 \pm 4$ \\
\hline
\end{tabular}

Diameters, polydispersity indexes (PDIs), and $\zeta$ potentials of carriers. (Data are means \pm S.D., $n=$ 3-5). Mitochondrial binding activities (\%) of carriers were measured using isolated mitochondria (Data presented are means \pm S.D., $\mathrm{n}=3-8$ ).

Dmt stands for 2, 6-dimethyltyrosine (SS, S2 ), X stands for cyclohexylalanine (MPP6). 


\title{
Supporting Information
}

\author{
Fig. S1
}

A

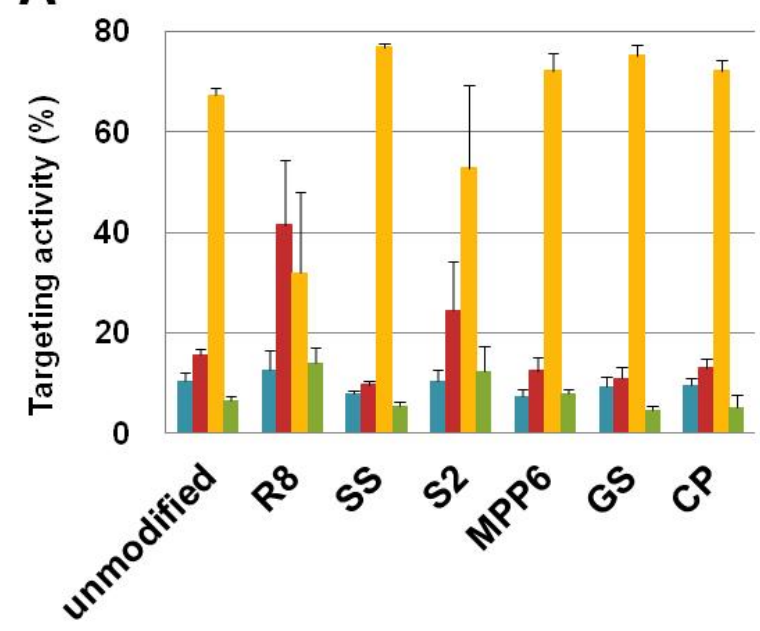

B

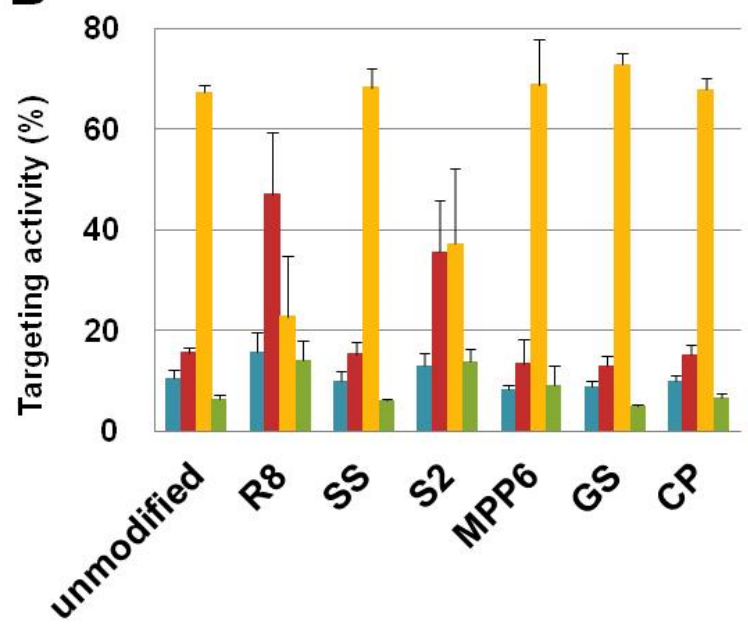

nucleus mitochondria $\square$ cytosol $\square$ other

Fig. S1. Organelle targeting activities of carriers. Targeting activity (\%) of 10\% (A), and 15\% (B) peptide modified liposomes in nuclear (blue bars), mitochondrial (red bars), cytosol (yellow bars) and other (green bars) fractions were evaluated. Data indicate the mean \pm S.D. $(n=3-5)$. 


\section{Table S1}

Characteristics of oligo DNA encapsulated carriers.

\begin{tabular}{lccc}
\hline & Diameter $(\mathrm{nm})$ & PDI & $\zeta$ potential $(\mathrm{mV})$ \\
\hline DF-S2-MITO-Porter & $179 \pm 14$ & $\mathbf{0 . 2 3 9} \pm \mathbf{0 . 1}$ & $\mathbf{3 8} \pm \mathbf{1 4}$ \\
DF-R8-MITO-Porter & $179 \pm 19$ & $\mathbf{0 . 1 9 7 \pm 0 . 0 7}$ & $52 \pm 5$ \\
\hline
\end{tabular}

PDI, polydispersity index. Data indicate means \pm S.D., $n=3-4$ 